Dental Considerations After the Outbreak of 2019 Novel Coronavirus

\title{
Disease: A Review of Literature
}

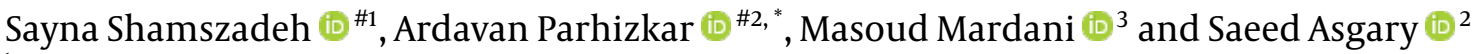 \\ ${ }^{1}$ Dental Research Centre, Research Institute of Dental Sciences, Shahid Beheshti University of Medical Science, Tehran, Iran \\ ${ }^{2}$ Iranian Center for Endodontic Research, Research Institute of Dental Sciences, Shahid Beheshti University of Medical Science, Tehran, Iran \\ ${ }^{3}$ Infectious Diseases and Tropical Medicine Research Center, Shahid Beheshti University of Medical Sciences, Tehran, Iran \\ "Corresponding author: Iranian Center for Endodontic Research, Research Institute of Dental Sciences, Shahid Beheshti University of Medical Science, Tehran, Iran. Email: \\ ardavanparhizkar@yahoo.co.uk \\ \# These authors are contributed equally as the first author.
}

Received 2020 March 31; Accepted 2020 April 02.

\begin{abstract}
In late December 2019, a new type of coronavirus, called novel coronavirus disease 2019 (COVID-19), caused a major outbreak of severe pneumonia in Wuhan (Huanan seafood market) and then, across China. As of 31 March 2020, COVID-19 has spread in 200 countries, including Iran, and imposed serious health-related threats to the public worldwide. Fever, dry (non-productive) cough, myalgia, and pneumonia seem to be the symptoms of COVID-19 disease. To date, and despite various studies and investigations, except for chloroquine and hydroxychloroquine, which have recently been temporarily approved by FDA, no other vaccines and/or antiviral agents have been clinically endorsed for the treatment of COVID-19 disease. Nevertheless, prevention of the disease and control of the infection is deemed a top priority for the general public. Owing to the characteristics of different dental treatments and settings, the risk of virus transmission can be considered high between patients and dental practitioners. Therefore, the need for strict and tough effective infection control protocols in dental practice is of great importance, namely for the dental centers in the potentially affected areas with COVID-19. This study, based on relevant evidence, aimed to review the basic knowledge of COVID-19 and address the recommended protocols of infection control for the dental practitioners and treatments in hypothetically stricken areas.
\end{abstract}

Keywords: Novel Coronavirus Disease 2019, COVID-19, Dentistry, Infection Control, Infectious Disease, Occupational Exposure, Protection

\section{Context}

In late December 2019, a cluster of infected patients with severe respiratory distress (pneumonia) of unknown etiology were reported in Wuhan, China (1). The viruscaused malady seriously afflicted the society and rapidly spread to other countries (2). In mid-January 2020, "World Health Organization" (WHO) named the virus severe respiratory syndrome-coronavirus-2 (SARS-COV-2), introducing it as the main trigger for acute respiratory distress syndrome (ARDS), and the malady was termed "coronavirus disease 2019 (COVID-19)". In late January, WHO declared a state of COVID-19 pandemic, and defined it as a public health emergency of global concern (3). Fortunately, 80\% of the involved cases show no clinical symptoms; however, in symptomatic cases, fever, fatigue (myalgia), dry coughs (without phlegm), and acute respiratory problems are considered to be the symptoms of the disease (4).

Owing to the characteristics of dental treatments and settings, the risk of cross-infection, including viral and bacterial pathogens between dental practitioners/personnel and patients, cannot be ignored (5). Despite vigorous ef- forts by researchers and scientists, as well as the commencement of related clinical studies, except for chloroquine and hydroxychloroquine, which have recently been granted the state of "Emergency Use Authorization (EUA)" by $\operatorname{FDA}(6,7)$, no other effective vaccines or medicinal drugs have been approved for the prevention and/or treatment of COVID-19 (8). Therefore, the need for strict and tough effective protocols for dental practices, especially in potentially affected areas with COVID-19, is of immense significance. In this study, COVID-19 is reviewed, and the recent protocols for infection control in dental treatments and corresponding centers are addressed.

\section{Novel Coronavirus Disease 2019 (COVID-19)}

Coronaviruses are defined as the largest group of viruses, which belong to the Nidovirales order, including Coronaviridae (alpha, beta, gamma, and delta), Arteriviridae, and Roniviridae families. These families are enveloped, non-segmented positive-sense RNA viruses. Coronaviruses can cause digestive and respiratory diseases in 
birds and mammals, as well as lethal respiratory infections in humans (9). COVID-2019 seems to be closely related to "Severe Acute Respiratory Syndrome" (SARS)-like coronaviruses (beta-coronavirus) (10). It has been shown that the structure of the receptor-binding domain (RBD) of COVID-19 is quite comparable with SARS coronavirus, and through the similar RBD of their spike protein, COVID-19 can enter the cell via human angiotensin-converting enzyme 2 (ACE2) receptor (11). Epidemiological findings on the first cases of COVID-19 infected patients revealed that the virus was primarily transmitted through bats (12), and pangolins (13) to humans, and then, it was spread personto-person (12).

\section{COVID-19: World and Iran}

As of 31 March 2020, it has been reported that COVID19 has spread and affected 200 countries and territories around the world. Overall, 799,724 individuals have tested positive for the virus, with 169,989 individuals recovered, and 38,721 individuals passed away worldwide. Moreover, and at the same date, the top ten countries which mostly suffered from COVID-19 were USA (164,359 positive cases; 3,173 total death), Italy (101,739 positive cases; 11,591 total death), Spain (94,417 positive cases; 8,189 total death), China (81,518 positive cases; 3,305 total death), Germany (67,051 positive cases; 650 total death), Iran (44,605 positive cases; 2,898 total death), France ( 44,550 positive cases; 3,024 total death), UK (22,141 positive cases; 1,408 total death), Switzerland (15,922 positive cases; 359 total death) and Belgium (12,775 positive cases; 705 total death)(14).

\section{Routes of Transmission}

Direct contact (person-to-person) and indirect contact (close contact less than 6 feet, inhaled droplets, aerosols, fecal-oral routes) are the major routes of transmission for COVID-19 (15-17). The incubation period of COVID-19 disease is usually long (varied from 3 - 14 days); however, it differs from one person to another (18). Asymptomatic patients and stealth carriers can transmit and infect other individuals (19).

\section{Infection Control in Dental Centers and Practices}

The widespread outbreak of COVID-19 (especially in Iran) and its incubation period (20) have made the prevention and control of infection very difficult. Since the main transmission route of the novel coronavirus 2019 is through respiratory droplets, aerosols, and direct contact
(21), dental practitioners and personnel are considered a high-risk group (15).

Since there are no vaccine and/or effective medicinal drugs for the prevention and treatment of COVID-19 (22), modifications in the management strategies on infection control protocols in dental centers and practices, particularly in the post-pandemic era, is of great significance. To help dental professionals/personnel to effectively combat the disease, taking into account the current investigations and evidence, some presented measures are as follows:

\subsection{General Recommendations}

\subsubsection{Patient Evaluation in the Waiting Room}

The waiting room in the dental practice/center should be sufficiently ventilated. Before the patient's entry to the dental chair-side, patients should be provided with a surgical or face mask and disinfectant gel, and their temperature should be measured using a sterile or contact-free forehead thermometer. Patients should stay at least six feet from one another/dental personnel. A thorough medical history should be obtained from the patients, and designated screening form (Figure 1) should be precisely completed and signed by the patients.

Furthermore, patients' education on sneeze and cough etiquettes is of immense importance and should be addressed as follows:

a. Patient encouragement to use disposable tissues and handkerchiefs during coughs and sneezes.

b. Disposal of tissues and handkerchiefs, immediately after use into a garbage bin.

c. Hand hygiene education, after coughs, sneezes, contact with respiratory droplets or contaminated items via mentioned secretions.

All surfaces in the dental practice environment and medical instruments (e.g., thermometers and sphygmomanometers) should be carefully and repeatedly disinfected using ethanol 70\% (23). If a patient is suspected to be positive for COVID-19, having symptoms related to the malady or positive answer to the screening questions, they should be immediately identified, quarantined and referred to the department of infection control in a hospital/center or the local health department (15). However, in case of urgent dental problems, patients can receive necessary treatments even if they are in the acute phase of the disease. In accordance with WHO, the suspicious and positive patients should be transferred to specific precaution rooms with negative pressure ventilation (12 air changes/hour "ACH" or $160 \mathrm{~L} / \mathrm{s} /$ patient) (24). Patients who do not report any symptoms, can receive dental care and treatments while considering all the above-mentioned advice (Figure 2). 
Date:

Full Name:

DOB:

\begin{tabular}{|l|l|l|}
\hline \multicolumn{1}{|c|}{ Screening Questions } & Affirmative & Negative \\
\hline $\begin{array}{l}\text { In the last 14 days, have any members of your family or a close friend of yours been } \\
\text { infected by COVID-19? }\end{array}$ & & \\
\hline $\begin{array}{l}\text { In the last 14 days, have you ever been in contact with a COVID-19 infected } \\
\text { patient? }\end{array}$ & & \\
\hline In the last 14 days, have you or any close persons been on a journey? & & \\
\hline In the last 14 days, have you had dry coughs or difficulty in breathing? & & \\
\hline In the last 14 days, have you had fever/temperature? & & \\
\hline
\end{tabular}

Figure 1. COVID-19 screening request form in dental practices

\subsubsection{Hand Hygiene}

Hand hygiene is one of the principal pathways for reducing the transfer of microorganisms to the patients (25). According to WHO, SARS-CoV-2 can survive on different surfaces from a number of hours to several days (26). Since oral-fecal transmission of the virus has been reported for COVID-19 (27), hand hygiene has become of utmost importance for dental professionals/personnel. Hand hygiene can be achieved in two ways; handwashing with water and soup and hand disinfection using alcohol-based solutions, both for 20 seconds.

In the following circumstances, dental professionals and personnel should follow hand hygiene protocols and prevent their hands from direct contact with eyes, nose, and mouth:

a. Before examination of patients, and beginning of dental treatment.

b. After contact with patients, environmental surfaces and materials/ substances contaminated with blood and body liquids/secretions.

c. After contact with secretions, mouth mucosa, and injured skin.

\subsubsection{Personal Protection Measures}

Aerosols, which are defined as small and tiny floating particles, are produced in most dental treatments (28, 29). Aerosols can stay suspended in the air for a long time
(30) and carry/transfer different substances, from blood and cells to restorative materials and microorganisms (31). Aerosols can be inhaled by dental professionals/personnel and cause respiratory infections and complications (32, 33). Therefore, using self-protective measures is highly recommended for dental ministrations.

a. Protective goggles and face shields

Evidence shows that ocular tissues are susceptible to the transmission of microparticles and aerosols (34). Thus, it is highly recommended to use protective goggles or face shields during dental treatments; so that the eyes and surrounding areas are perfectly covered and protected from the entry of microorganisms. After termination of a dental treatment, the goggle/shield should be properly washed and completely disinfected.

b. Face masks

After termination of dental treatments, used face masks must be disposed. Surgical masks are recommended when the operator is at a distance of less than six feet from the patient, and special masks, i.e., N95 respirator and FFP2 masks are suggested when there is a possibility of aerosol production.

c. Protective outwear (gown)

Protective outwears should be changed after the termination of each dental treatment.

d. Gloves

Dental professionals and personnel should change 


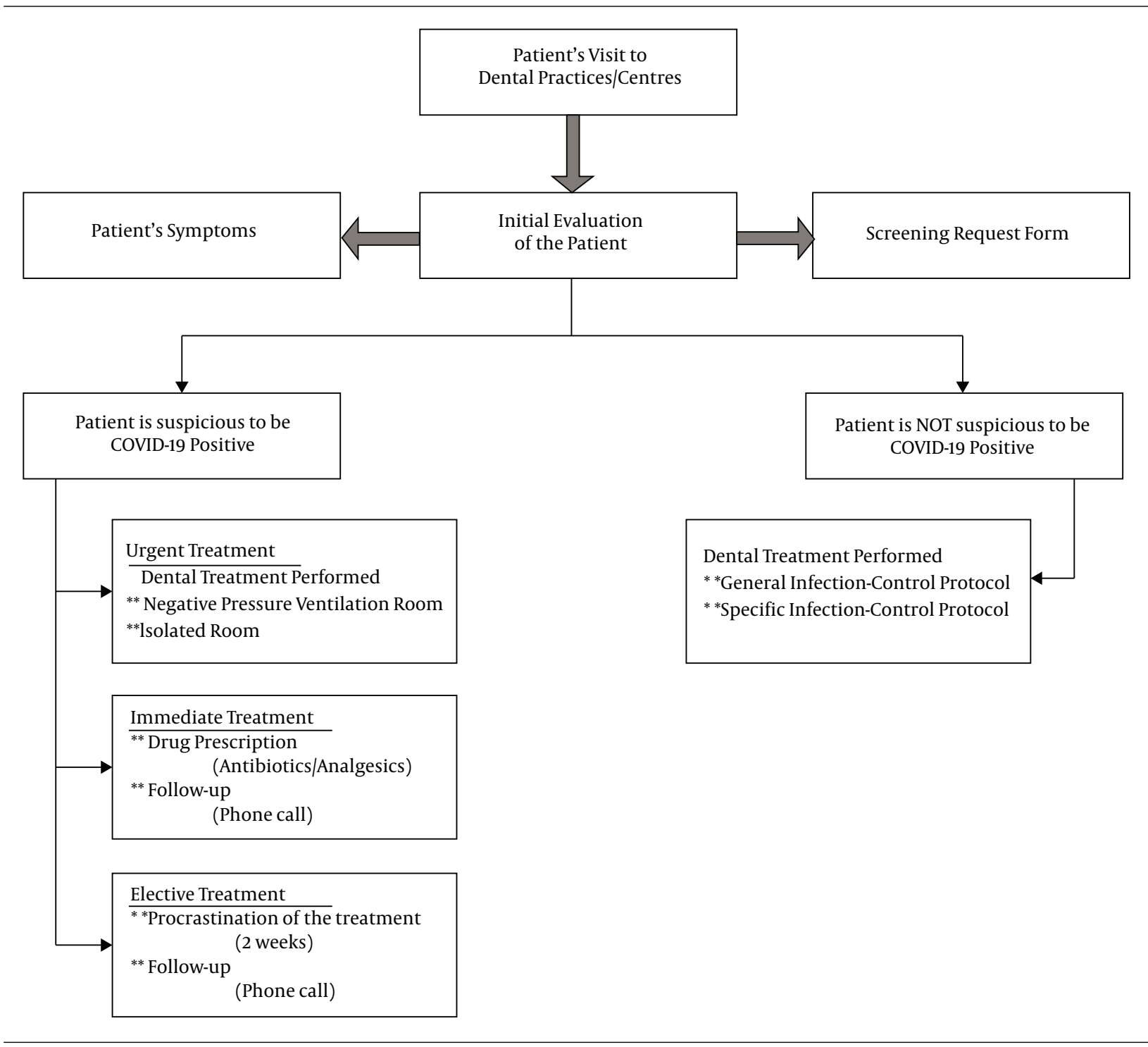

Figure 2. Dental treatment protocols after the outbreak of COVID-19

their protective gloves (latex, nitrile, nylon, and so forth) after the termination of each dental treatment. In addition, hand hygiene protocols should be precisely followed immediately before and after wearing gloves. Protective gloves might have unidentified or small tears, which could end in rupture of the glove during use. It is highly recommended to use two pairs of gloves during dental surgical procedures.

\subsubsection{Surface Disinfection}

Dental chair-side surfaces -i.e., dental light handle, dental chair keyboard keys, connected computers/laptops, oral cameras, dental surgery drawer handles, water taps, stationaries, working desks, telephone sets, doorknobs and so forth-and peripheral surfaces-i.e., floor, walls, washing sink/faucets and so on- should be disinfected in the intervals between patients using ethanol 70\%.

\subsubsection{Mouth-Rinsing Before Dental Treatment}

Mouth-rinsing with antibacterial solutions cannot impede the transfer of COVID-19 from the patients to the dental professionals/personnel and vice versa; nevertheless, it can reduce the number of microorganisms, which are in contact with blood during invasive dental treatments (35).

Using mouth-rinses before dental treatments can cause a reduction in the oral cavity microorganisms (3638). In accordance with the guidelines in the diagnosis and treatment of COVID-19-caused pneumonia by the health 
council of the People's Republic of China, chlorhexidine (as a common mouth-rinse and mouthwash solution) may not be effective against COVID-19. Since the virus is quite sensitive to oxidation, using a mouth rinse or mouthwash solutions containing hydrogen peroxide is recommended (39).

\subsubsection{Use of Other Materials/Instruments/Equipment}

Rubber dam, specifically when handpieces and ultrasonic devices are used, can minimize the dispersion of droplets, secretions, and aerosols. It is recommended to use high volume evacuators (HVE) when rubber dam is to be applied. In these circumstances, four-handed technique is usually practiced. If rubber dam cannot be applied, the use of hand instruments, e.g., hand scalers/curettes are recommended for periodontal purposes or carisolv for chemomechanical caries removal (40).

\subsubsection{Removal of Medical/Dental Waste}

Disposable protective equipment should be transferred to a temporary storage area. The medical waste -from the treatment of patients suspicious to COVID-19should be considered an infectious residue. These wastes should be packed in two-layered packages and sealed properly.

\subsection{Specific Dental Considerations for the Reduction of Aerosols}

In all branches of dentistry, the mentioned infectioncontrol principles should be carefully followed. However, in a number of specialties, there are other points to consider:

\subsubsection{Endodontics}

The application of rubber dam in endodontic treatments is highly recommended at all times. In these circumstances, it is advocated to use hand instruments instead of rotary systems. In the case of pain due to symptomatic irreversible pulpitis, pulp exposure and devitalization could be made with chemomechanical caries removal and a HVE after local anesthesia under rubber dam isolation. In these circumstances, vital pulp therapy (VPT) is much preferred to conventional root canal treatments.

\subsubsection{Restorative and Pediatric Dentistry}

In elective cases, high treatment priority is given to chemomechanical caries removal and atraumatic restorative techniques. Similar to endodontic considerations, hand instrumentation for cavity preparation is preferred to rotary preparations. However, if rotary instruments are to be considered, rubber dam isolation should pertain. Moreover, manual scaling and polishing are endorsed.

\subsubsection{Periodontics}

It seems that hand and ultrasonic approaches have similar clinical effects on the reduction of dental plaque and calculi (41), and there were no significant differences between the two methods $(42,43)$. Therefore, and in suspicious circumstances, using instruments manually is preferable.

\subsubsection{Prosthodontics}

To avoid gagging in patients, salivary suction should be used. If an impression is taken, the impression and other prosthodontic materials (e.g., bite registration and so on) should be properly disinfected prior to the delivery to prosthodontic laboratories. The disinfection procedure is as follows:

a. Initial irrigation using tap water

b. Placement of the material in a disinfectant with at least intermediate level activity, i.e., sodium hypochlorite for 10 minutes.

c. Re-irrigation using tap water

Furthermore, the application of rubber dam is an alltime necessity for crown and bridge/other prosthodontic preparations. Modification of treatment plans can also be considered; for example, supra-gingival margins for posterior bridges or using split-dam techniques.

\subsubsection{Oral and Maxillofacial Radiology}

Extraoral dental radiographies i.e., cone beam computed tomography (CBCT) and panoramic radiography are recommended.

\subsubsection{Oral and Maxillofacial Surgery}

When ministrations e.g., a simple extraction, are performed, the patient should be treated in the supine position. Using salivary suction and/or HVE during procedures is necessary. When there is a wound, it should be rinsed slowly. In the case of sutures, using absorbable material is preferable.

\section{Conclusions}

The widespread outbreak of COVID-19 and the pandemic of the novel coronavirus have become a global concern and a serious threat to the health, well-being, and survival of humans. The characteristics of dental treatments and the possibility of aerosol production during such ministrations classify dentistry as one of the most high-risk jobs in the world. The existing knowledge on COVID19 is currently limited, and studies for vaccines, effective medicinal drugs, and possible treatments are imminent. Comprehensive research and investigations are needed 
to clarify COVID-19 routes of transmission, pathogenicity, and its mechanisms, and define potential pharmaceutical purposes. Hereby, we addressed protocols on infectioncontrol for general and specific dental treatments and presented conventions for the protection of dental professionals/personnel from the transmission of the virus in the coming months.

\section{Footnotes}

Authors' Contribution: Study concept and design: Ardavan Parhizkar, Sayna Shamszadeh, and Saeed Asgary; analysis and interpretation of data: Ardavan Parhizkar, Sayna Shamszadeh, Saeed Asgary, and Masoud Mardani; acquisition of data: Ardavan Parhizkar, Sayna Shamszadeh, Saeed Asgary, and Masoud Mardani; administrative, technical, and material support: Ardavan Parhizkar, Sayna Shamszadeh, and Saeed Asgary; project administration: Ardavan Parhizkar, Sayna Shamszadeh, and Saeed Asgary; study supervision: Saeed Asgary; writing-drafting the manuscript: Ardavan Parhizkar; Sayna Shamszadeh, and Saeed Asgary; writing-critical revision of the manuscript for important intellectual content: Ardavan Parhizkar, Sayna Shamszadeh, Saeed Asgary, and Masoud Mardani

Conflict of Interests: The authors declare that there is no conflict of interest regarding the publication of this paper.

Funding/Support: The authors declared that no funding/financial support was received regarding this paper.

\section{References}

1. Zhu N, Zhang D, Wang W, Li X, Yang B, Song J, et al. A novel coronavirus from patients with pneumonia in China, 2019. N Engl J Med. 2020;382(8):727-33. doi: 10.1056/NEJMoa2001017. [PubMed: 31978945]. [PubMed Central: PMC7092803].

2. Wang C, Horby PW, Hayden FG, Gao GF. A novel coronavirus outbreak of global health concern. Lancet. 2020;395(10223):470-3. doi: 10.1016/S0140-6736(20)30185-9. [PubMed: 31986257]. [PubMed Central: PMC7135038].

3. Wong J, Goh QY, Tan Z, Lie SA, Tay YC, Ng SY, et al. Preparing for a COVID19 pandemic: A review of operating room outbreak response measures in a large tertiary hospital in Singapore. Can J Anaesth. 2020. doi: 10.1007/s12630-020-01620-9. [PubMed: 32162212]. [PubMed Central: PMC7090449].

4. Huang C, Wang Y, Li X, Ren L, Zhao J, Hu Y, et al. Clinical features of patients infected with 2019 novel coronavirus in Wuhan, China. Lancet. 2020;395(10223):497-506. doi: 10.1016/S0140-6736(20)30183-5. [PubMed: 31986264].

5. Volgenant CMC, de Soet JJ. Cross-transmission in the dental office: Does this make you ill? Curr Oral Health Rep. 2018;5(4):221-8. doi: 10.1007/s40496-018-0201-3. [PubMed: 30524929]. [PubMed Central: PMC6244620].

6. Hinton DM. Final hydroxychloroquine and chloroquine LOA. Food and Drug Administration; 2020

7. HHS. HHS accepts donations of medicine to Strategic National Stockpile as possible treatments for COVID-19 patients. 2020. Available from: https://www.hhs.gov/about/news/2020/03/29/hhs- accepts-donations-of-medicine-to-strategic-national-stockpile-aspossible-treatments-for-covid-19-patients.html.

8. Prompetchara E, Ketloy C, Palaga T. Immune responses in COVID-19 and potential vaccines: Lessons learned from SARS and MERS epidemic. Asian Pac J Allergy Immunol. 2020;38(1):1-9. doi: 10.12932/AP200220-0772. [PubMed: 32105090].

9. Fehr AR, Perlman S. Coronaviruses: An overview of their replication and pathogenesis. Methods Mol Biol. 2015;1282:1-23. doi: 10.1007/978-14939-2438-7_1. [PubMed: 25720466]. [PubMed Central: PMC4369385].

10. Wu F, Zhao S, Yu B, Chen YM, Wang W, Song ZG, et al. A new coronavirus associated with human respiratory disease in China. $\mathrm{Na}$ ture. 2020;579(7798):265-9. doi: 10.1038/s41586-020-2008-3. [PubMed: 32015508]. [PubMed Central: PMC7094943].

11. Hu B, Zeng LP, Yang XL, Ge XY, Zhang W, Li B, et al. Discovery of a rich gene pool of bat SARS-related coronaviruses provides new insights into the origin of SARS coronavirus. PLoS Pathog. 2017;13(11). e1006698. doi: 10.1371/journal.ppat.1006698. [PubMed: 29190287]. [PubMed Central: PMC5708621].

12. Chan JF, Yuan S, Kok KH, To KK, Chu H, Yang J, et al. A familial cluster of pneumonia associated with the 2019 novel coronavirus indicating person-to-person transmission: A study of a family cluster. Lancet. 2020;395(10223):514-23. doi: 10.1016/S0140-6736(20)30154-9. [PubMed: 31986261].

13. Lam TT, Shum MH, Zhu HC, Tong YG, Ni XB, Liao YS, et al. Identifying SARS-CoV-2 related coronaviruses in Malayan pangolins. Nature. 2020. doi: 10.1038/s41586-020-2169-0. [PubMed: 32218527].

14. Worldometer. Countries where COVID-19 has spread. 2020. Available from: https://www.worldometers.info/coronavirus/countrieswhere-coronavirus-has-spread/.

15. Peng X, Xu X, Li Y, Cheng L, Zhou X, Ren B. Transmission routes of 2019-nCoV and controls in dental practice. Int J Oral Sci. 2020;12(1):9. doi: 10.1038/s41368-020-0075-9. [PubMed: 32127517]. [PubMed Central: PMC7054527].

16. Xu H, Zhong L, Deng J, Peng J, Dan H, Zeng X, et al. High expression of ACE2 receptor of 2019-nCoV on the epithelial cells of oral mucosa. Int J Oral Sci. 2020;12(1):8. doi: 10.1038/s41368-020-0074-x. [PubMed: 32094336]. [PubMed Central: PMC7039956].

17. Lu CW, Liu XF, Jia ZF. 2019-nCoV transmission through the ocular surface must not be ignored. Lancet. 2020;395(10224). e39. doi: 10.1016/S0140-6736(20)30313-5. [PubMed:32035510]. [PubMed Central: PMC7133551].

18. Backer JA, Klinkenberg D, Wallinga J. Incubation period of 2019 novel coronavirus (2019-nCoV) infections among travellers from Wuhan, China, 20-28 January 2020. Euro Surveill.2020;25(5). doi: 10.2807/15607917.ES.2020.25.5.2000062. [PubMed: 32046819]. [PubMed Central: PMC7014672].

19. Shi Y, Wang Y, Shao C, Huang J, Gan J, Huang X, et al. COVID-19 infection: The perspectives on immune responses. Cell Death Differ. 2020. doi: 10.1038/s41418-020-0530-3. [PubMed: 32205856].

20. Lauer SA, Grantz KH, Bi Q, Jones FK, Zheng Q, Meredith HR, et al. The incubation period of coronavirus disease 2019 (COVID-19) from publicly reported confirmed cases: Estimation and application. Ann Intern Med. 2020. doi: 10.7326/M20-0504. [PubMed: 32150748]. [PubMed Central: PMC7081172].

21. Zhou W, Zhong N. The coronavirus prevention handbook: 101 sciencebased tips that could save your life. 2020, [cited 2020 Mar 26].

22. Dong L, Hu S, Gao J. Discovering drugs to treat coronavirus disease 2019 (COVID-19). Drug Discov Ther. 2020;14(1):58-60. doi: 10.5582/ddt.2020.01012. [PubMed: 32147628].

23. Cascella M, Rajnik M, Cuomo A, Dulebohn SC, Di Napoli R. Features, evaluation and treatment coronavirus (COVID-19). StatPearls. Treasure Island (FL); 2020. eng.

24. Chartier Y, Pessoa-Silva CL. Natural ventilation for infection control in health-care settings. World Health Organization; 2009. 
25. Larson EL, Early E, Cloonan P, Sugrue S, Parides M. An organizational climate intervention associated with increased handwashing and decreased nosocomial infections. Behav Med. 2000;26(1):14-22. doi: 10.1080/08964280009595749. [PubMed: 10971880].

26. World Health Organization. Questions and answers on coronaviruses. 2020, [cited 2020 Feb 26]. Available from: https://www.who.int/ news-room/q-a-detail/q-a-coronaviruses.

27. Yuen KS, Ye ZW, Fung SY, Chan CP, Jin DY. SARS-CoV-2 and COVID-19: The most important research questions. Cell Biosci. 2020;10:40. doi: 10.1186/s13578-020-00404-4. [PubMed: 32190290]. [PubMed Central: PMC7074995].

28. Sawhney A, Venugopal S, Babu GR, Garg A, Mathew M, Yadav M, et al. Aerosols how dangerous they are in clinical practice. J Clin Diagn Res. 2015;9(4):ZC52-7. doi: 10.7860/JCDR/2015/12038.5835. [PubMed: 26023644]. [PubMed Central: PMC4437160].

29. Kobza J, Pastuszka JS, Bragoszewska E. Do exposures to aerosols pose a risk to dental professionals? Occup Med (Lond). 2018;68(7):454-8. doi: 10.1093/occmed/kqy095. [PubMed: 29931138]. [PubMed Central: PMC6135984].

30. Centers for Disease Control and Prevention. Healthcare-associated infections. 1999. Available from: http://www.cdc.gov/ncidod/dhqp/bp_ hiv_aerosol.html.

31. Feres M, Figueiredo LC, Faveri M, Stewart B, de Vizio W. The effectiveness of a preprocedural mouthrinse containing cetylpyridinium chloride in reducing bacteria in the dental office. J Am Dent Assoc. 2010;141(4):415-22. doi: 10.14219/jada.archive.2010.0193. [PubMed: 20354090].

32. Yamada H, Ishihama K, Yasuda K, Hasumi-Nakayama Y, Shimoji S, Furusawa K. Aerial dispersal of blood-contaminated aerosols during dental procedures. Quintessence Int. 2011;42(5):399-405. [PubMed: 21519559].

33. Mikitka D, Mills SE, Dazey SE, Gabriel ME. Tuberculosis infection in U.S. Air Force dentists. Am J Dent. 1995;8(1):33-6. [PubMed: 7546471].

34. Belser JA, Gustin KM, Katz JM, Maines TR, Tumpey TM. Influenza virus infectivity and virulence following ocular-only aerosol inoculation of ferrets. J Virol. 2014;88(17):9647-54. doi: 10.1128/JVI.01067-14. [PubMed: 24920819]. [PubMed Central: PMC4136304].

35. Fine DH, Furgang D, Korik I, Olshan A, Barnett ML, Vincent JW. Reduction of viable bacteria in dental aerosols by preprocedural rinsing with an antiseptic mouthrinse. Am J Dent. 1993;6(5):219-21. [PubMed:
7880461].

36. Retamal-Valdes B, Soares GM, Stewart B, Figueiredo LC, Faveri M, Miller S, et al. Effectiveness of a pre-procedural mouthwash in reducing bacteria in dental aerosols: Randomized clinical trial. Braz Oral Res. 2017;31. e21. doi: 10.1590/1807-3107BOR-2017.vol31.0021. [PubMed: 28380086].

37. Santos IR, Moreira AC, Costa MG, Castellucci e Barbosa M. Effect of $0.12 \%$ chlorhexidine in reducing microorganisms found in aerosol used for dental prophylaxis of patients submitted to fixed orthodontic treatment. Dental Press J Orthod. 2014;19(3):95-101. doi: 10.1590/2176-9451.19.3.095-101.0ar. [PubMed: 25162572]. [PubMed Central: PMC4296627].

38. Hunter A, Kalathingal S, Shrout M, Plummer K, Looney S. The effectiveness of a pre-procedural mouthrinse in reducing bacteria on radiographic phosphor plates. Imaging Sci Dent. 2014;44(2):149-54. doi: 10.5624/isd.2014.44.2.149. [PubMed: 24944965]. [PubMed Central: PMC4061299].

39. Jin YH, Cai L, Cheng ZS, Cheng H, Deng T, Fan YP, et al. A rapid advice guideline for the diagnosis and treatment of 2019 novel coronavirus (2019-nCoV) infected pneumonia (standard version). Mil Med Res. 2020;7(1):4. doi: 10.1186/s40779-020-0233-6. [PubMed: 32029004]. [PubMed Central: PMC7003341].

40. Kathuria V, Ankola AV, Hebbal M, Mocherla M. Carisolv- an innovative method of caries removal. J Clin Diagn Res. 2013;7(12):3111-5. doi: 10.7860/JCDR/2013/6676.3873. [PubMed: 24551746]. [PubMed Central: PMC3919277].

41. Chapper A, Catao VV, Oppermann RV. Hand and ultrasonic instrumentation in the treatment of chronic periodontitis after supragingival plaque control. Braz Oral Res. 2005;19(1):41-6. doi: 10.1590/s180683242005000100008. [PubMed: 16229355].

42. Zafar MS. Comparing the effects of manual and ultrasonic instrumentation on root surface mechanical properties. Eur J Dent. 2016;10(4):517-21. doi: 10.4103/1305-7456.195162. [PubMed: 28042268]. [PubMed Central: PMC5166309].

43. Amid R, Kadkhodazadeh M, Fekrazad R, Hajizadeh F, Ghafoori A. Comparison of the effect of hand instruments, an ultrasonic scaler, and an erbium-doped yttrium aluminium garnet laser on root surface roughness of teeth with periodontitis: A profilometer study. $\mathrm{J} \mathrm{Pe}$ riodontal Implant Sci. 2013;43(2):101-5. doi: 10.5051/jpis.2013.43.2.101. [PubMed: 23678394]. [PubMed Central: PMC3651935]. 